\title{
Development of Computerized Human Static Strength Simulation Model for Job Design
}

\author{
Don B. Chaffin \\ Industrial and Operations Engineering, 1205 Beal Avenue, \\ The University of Michigan, Ann Arbor, MI 48109-2117
}

\begin{abstract}
This article describes the development of models to predict population static strengths and low back forces resulting from common manual exertions in industry. The resulting biomechanical models are shown to be valid for their intended purposes, but limitations still exist. In particular, they are meant to aid in evaluating very slow or static exertions, such as when carefully lifting, pushing, or pulling on heavy objects, but do not allow dynamic exertions to be simulated. It is shown that use of these models in the early design of workplaces and equipment is dependent on the use of computerized homonoids and behavioral-based inverse kinematic algorithms in conjunction with CAD systems. (C) 1997 John Wiley \& Sons, Inc.
\end{abstract}

\section{INTRODUCTION-THE PROBLEM}

Manual exertions remain an essential part of many jobs today. Though the frequency of such exertions may not be as great now as several decades ago, thanks to mechanization and automation, many jobs still require occasional exertions of a high magnitude. These jobs are classified as ones that have a high worker strength demand. In 1981 it was estimated that one third of jobs in the United States contained manual exertions of this type (i.e., ones with significant strength requirements) (National Institute for Occupational Safety and Health [NIOSH], 1981). It is believed that these types of exertions still account for a disproportionate number of serious injuries, mostly to the musculoskeletal system. Based on the latest Department of Labor Survey of work-related injuries and illnesses, overexertion accounted for 27\% of injuries and illnesses in 1994 (U.S. Department of Labor, Bureau of Labor Statistics [USDL], 1996). The direct medical and wage replacement costs associated with these injuries and illnesses are estimated between $\$ 15$ and $\$ 20$ billion annually, with administrative and other indirect costs possibly quadrupling the total costs.

It also must be recognized that when a job includes manual exertions of a high magnitude, it is a job that becomes difficult to staff, due to the large variability that exists in a working populations' strength performance capability. In fact, a normal, healthy, mixed gender population of workers may have strength variations greater than 10:1 (Chaffin and Andersson, 1991). With implementation of the American's with Disabilities Act, this normal variation in population strength capabilities will increase in the working population. If a manual task in a job is found to be particularly difficult for older workers, women or physically impaired individuals, legal remedies may mandate changes in the job to elim- 
inate the discriminatory task, particularly if it is not an essential function in the job. For instance, an occasional stock loading task may be found to be secondary to the primary tasks required in operating a machine, and thus a mechanized loader may need to be purchased to meet court dictated affirmative action requirements to allow a person with limited strengths to perform the job. Thus, it is becoming imperative that in producing a professional job design the designer must anticipate not only the time required for a worker to perform the job, but also high exertion requirements (i.e., strength requirements) that may cause injury or discriminate against older workers (over 40 years), women, and physically impaired workers.

Unfortunately, traditional work measurement systems do not provide this information to designers, as they are meant to predict the average time to perform a task rather than to identify physiological and biomechanical demands (Chaffin and Andersson, 1991). This methodological limitation has been well recognized in the ergonomics literature over the last two decades, and has spawned a new set of job evaluation methods. The appropriateness and effectiveness of these new methods are now being determined for specific applications.

In this context, the advent of computerized human exertion simulation methods is of particular interest, in that they may be able to assist in evaluating manual exertions in jobs early in the design phase. Yet there is a tendency for those not aware of the complexity and variety of human-hardware physical interactions in industry to rely on "nice looking" computer-generated human form images in their CAD software as being reality. Unfortunately, the actual biomechanical stresses required by an individual may not be properly evaluated or presented. The situation today allows job designers, who are working on CAD systems, to purchase licenses to use a variety of human image software. These include SAMMIE (Porter et al., 1995), APOLIN (Grobelny et al., 1992), CAAA (Hoekstra, 1993), COMBIMAN and Crew Chief (McDaniel, 1990), Deneb/ERGO (Nayar, 1995), ERGOMAN (Mollard et al., 1992), ErgoSPACE (Launis and Lehtala, 1990), JACK (Badler, Becket, and Webber, 1995), TADAPS (Westerink et al., 1990), and SAFEWORK (Fortin et al., 1990) to name a few. Some of these programs render quite lifelike solid and shaded human forms, whereas others use a variety of simple silhouettes or wire frame images. In fact, one of the more technically challenging graphic problems in the past was to render the human body for computer presentation in a form that depicted the shape changes "smoothly" at each major joint of the body as the simulated person is moved into various postures by the job designer. In general, with the advent of newer, higher speed and graphic software most human images generated now don't look too "ragged" when placed in different postures, particularly if rendered on a graphic workstation.

One of the major issues today in human image generation is to provide an easy to use input method to move the computer-generated hominoid into a variety of normal postures chosen by individuals when performing a manual task. Past models have used simple robotic algorithms and inverse kinematic structures to chose initial postures. If the user doesn't like such a posture, a menu of joint angles is provided that allows the user to modify by direct keyboard entry a specific joint angle. On some programs, moving a cursor over a joint, clicking a mouse, and then moving the cursor to a new location provides a means to change a posture. Still, these methods rely heavily on the user knowing from experience how a person would normally stand, sit, bend, or stoop while performing a particular task. Such an approach often results in problematic postures being specified, especially when the user is far different anthropometrically or in work experience than the 
person expected to perform the task. Needless to say, this aspect of human posture simulation remains a major challenge today, and will be further discussed later in this article.

As stated earlier, providing computer-rendered human forms that allow the job designer to assess whether people of various size can reach an object and/or see an object is only part of the challenge today. If the job task being simulated also requires the operator to manually lift, push, or pull on an object, then a biomechanical strength assessment must be performed. What follows is a description of how one type of computerized strength simulation modeling has developed, and how it is being used to improve the design of manual high exertion tasks. The two major issues addressed are (1) how valid are computerized strength prediction models, and (2) how can they be configured within computeraided job design software to allow a user to easily assess a manual exertion?

\section{OVERVIEW OF BIOMECHANICAL STRENGTH PREDICTION MODELLING}

The general logic used to predict population static strengths in various jobs is depicted in Figure 1. In this model specific muscle group strength data and spinal vertebrae failure data are used as the limiting values for the reactive moments at various body joints created when a person of a designated stature and body weight attempts an exertion (i.e., lifts, pushes, or pulls in a specific direction with one or both hands while maintaining a known posture).

To compute the different joint moment loads simple Newtonian mechanics are used, wherein forces (i.e., body segment weights and hand loads) are multiplied by their perpendicular distance from joint centers (i.e., moment arms). The following is an example for the elbow moment load ME while holding a weight. Note in Figure 2 that the only three input data required are:

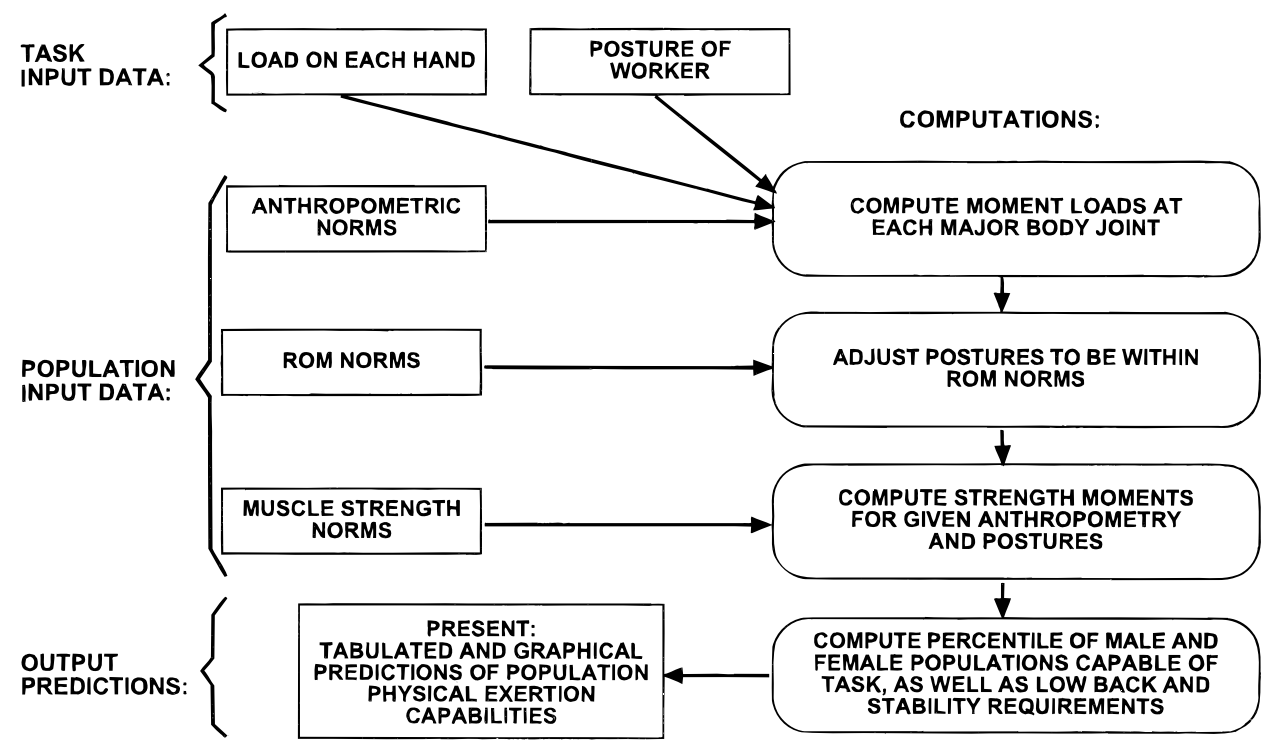

Figure 1 Biomechanical logic used to predict whole-body static exertion capabilities for given postures, hand force directions, and anthropometric groups. 


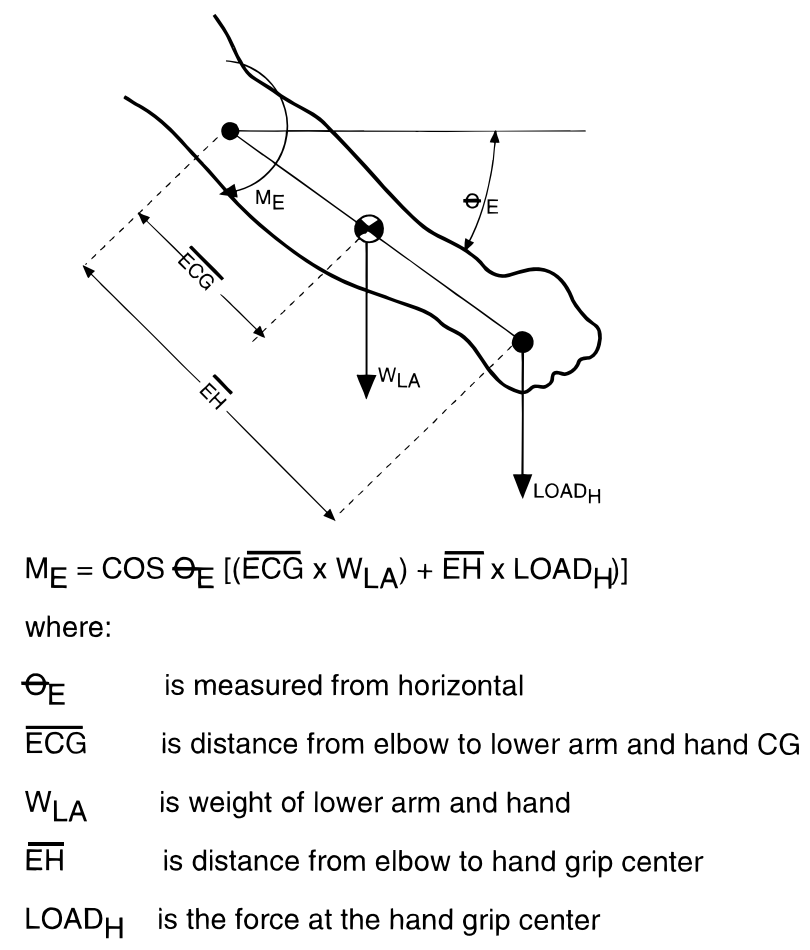

Figure 2 Elbow moment estimating procedure.

1. Hand forces $\left(\operatorname{Load}_{\mathrm{H}}\right)$

2. Anthropometry, which provides segment dimensions and weight.

3. Postures, which provide joint angle data.

A recursive method of computation is used to compute the moments at all of the major joints as shown in Figure 3. This logic has been well described for a sagittal, coplanar static strength analysis in Chaffin and Andersson (1991), and for three-dimensional exertions in Chaffin and Erig (1991). When wishing to perform an analysis in three dimensions, the body is represented as a set of links with known mass, as depicted in Figure 3. The load moments $M_{j}$ are computed by the cross products of the unit distance vectors to each joint and body segment weights and hand forces.

The size and mass of the person (linkage) is most often specified as a select strata of the population (i.e., a percentile of specific anthropometric dimensions is selected by the users from population surveys). Thus, a small, medium, or large man or woman can be specified, or specific link anthropometry can be used if available. Link length-to-stature ratios from Drillis and Contini (1966) and Link mass-to-body weight ratios from Dempster (1955) and Clauser, McConville, and Young (1969) are used to simplify this procedure if specific anthropometry is not available. Most often average (50th percentile) male or female anthropometry is chosen for assessing the strength requirements of a given task in industry, though any percentile representation can be used.

Once the joint load moments $M_{j}$ have been computed for a given exertion of interest, these are compared to population strength moments $S_{j}$ values. One of the first models to 

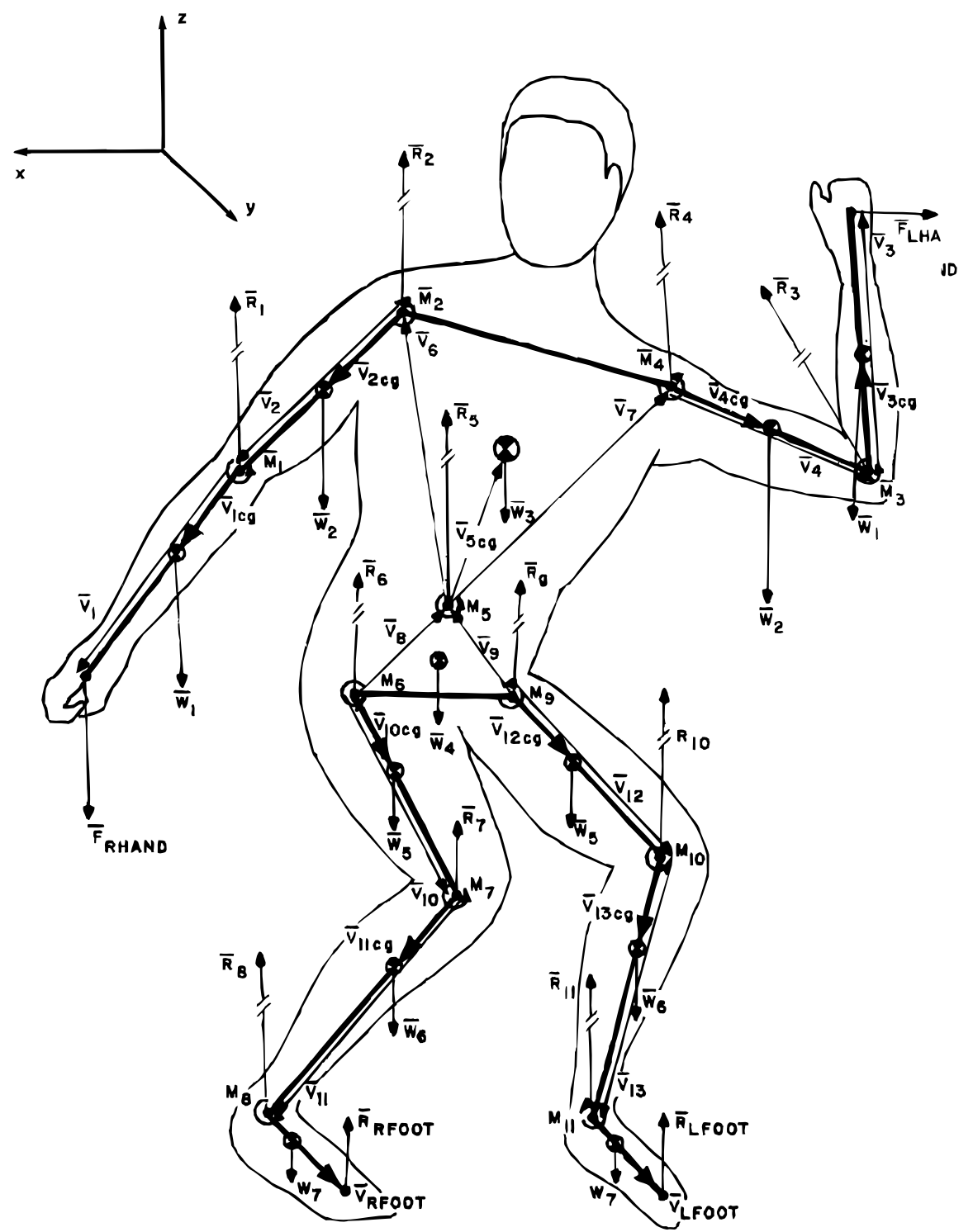

Figure 3 Three-dimensional distance, force, and moment vectors used in 12-link biomechanical model for strength prediction (Chaffin and Erig, 1991).

perform such a comparison was developed by Chaffin (1969) for analysis of load lifting activities in industry performed in the sagittal plane. In this early static strength prediction model the load moments $M_{j}$ values for given loads $L$ were compared to the flexion and extension strength $S_{j}$ at six major body joints, (i.e., elbow, shoulder, lumbosacral, hip, knee, and ankle joints) of the body, as follows:

$$
-S_{j}<M_{j} / L<S_{j}
$$


where $S_{j}=$ the muscle-produced moment strengths at each joint $j$ (with negative limits being clockwise and positive values being counterclockwise), and $M_{j} / L=$ the reactive moments acting at each joint $j$ due to external loads $L$ on the hands.

The $M_{j} / L$ values are computed from the application of static equilibrium conditions that produce estimates of the moments at each joint for given postures, anthropometry, and external loads as described earlier. The joint moment strengths $S_{j}$ are obtained by population measurements using standardized strength testing methods. Population values have been developed for most major muscle functions. In this latter regard, it must be recalled that muscle strength moments vary over the range of motion of a joint. Thus joint angles must be known to predict $S_{j}$ values. In fact, because muscles often span two joints, the angle at adjacent joints must be considered in predicting $S_{j}$ values. A synthesis of values for $S_{j}$ from the moment strength studies of Burggraaf (1972), Clarke (1966), and Schanne (1972) is presented in Chaffin and Andersson (1991).

Because the computations needed to estimate both the $M_{j}$ and $S_{j}$ values are intense, computer programs were developed for this purpose. The sagittal plane strength prediction program just described was first programmed in 1968 by Chaffin (1969) and was used to evaluate lunar exploration manual task simulations for NASA in the early 1970s (Martin and Chaffin, 1972). The resulting program is currently referred to as the "2D Static Strength Prediction Program"TM. A three-dimensional version, referred to as the "3D Static Strength Prediction Program"TM was programmed by Garg and Chaffin (1975). It compares the population strength capabilities with 24 different muscle joint strengths, and allows asymmetric exertions involving one or both hands to be evaluated.

\section{OVERVIEW OF LOW BACK STRENGTH MODELING}

From a biomechanical perspective, the fact that large moments are created at the lumbar spine when heavy loads are lifted raises the question of the nature of the internal forces that must be present to stabilize the spine when it incurs such large load moments. An early static, sagittal plane model of the lumbar spine during lifting was proposed by Morris, Lucas, and Bressler (1961). This model assumed that two types of internal forces act to provide the major resistance to the external load moment. One is the posterior erector spinae muscles, which at that time were believed to exert their effective force approximately $5 \mathrm{~cm}$ posterior to the centers-of-rotation of the spinal discs. The second major stabilizing force was assumed to be caused by abdominal pressure acting in front of the spinal column, pushing the upper torso into extension, and thus resisting the flexor load moment acting on the lumbar spine. From application of this type of model it was realized that large forces develop in the spinal column to compress the discs during common load-lifting acts. The general magnitude of these compression forces was later confirmed by Nachemson and Elfstrom (1970) in a series of experiments in which the pressure within the center portion of the discs was measured (by inserting a needle into the spinal discs attached to a pressure transducer) in volunteers who performed various light load lifting maneuvers.

An illustration of this type of model is shown in Figure 4, as adopted by Chaffin (1975) to include both a method for predicting the abdominal pressure from torso angle and torso moment loads, and a method of predicting the rotation of the pelvis as a function of torso and leg postures. The logic is explained and illustrated in Chaffin and Andersson (1991). This type of coplanar model has been imbedded in dynamic models used by Ayoub and El-Bassoussi (1978), Freivalds et al. (1984), and Leskinen et al. (1983) for analysis of lifting stresses. 


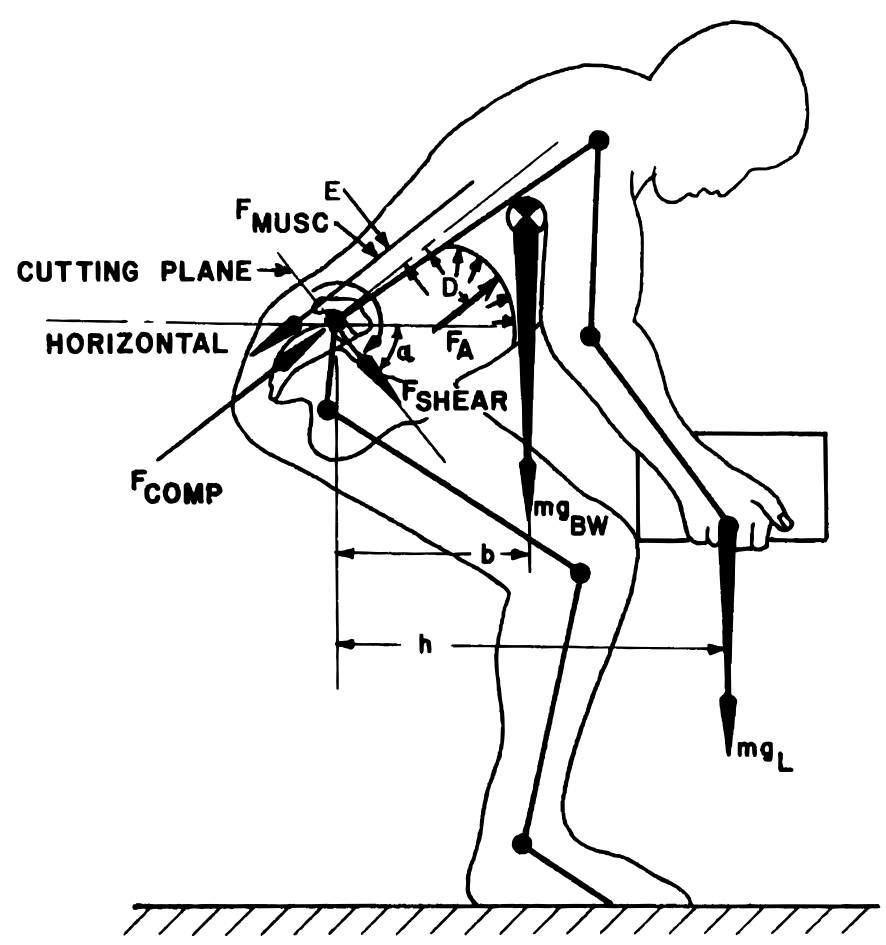

Figure 4 Simple cantilever low-back model of lifting as adapted by Chaffin (1975) for static coplanar lifting analyses (Chaffin and Andersson, 1991).

When the predicted compression forces from the models were compared to cadaverbased vertebrae cartilage endplate failure data, it was realized that many people could be at risk in the workplace when lifting moderate to heavy loads in certain postures. A summary of the cadaver, spinal compression failure forces is shown in Figure 5 from Jäger (1987).

More recent cadaver studies by Adams and Dolan (1995) indicate that the failure values are even lower with spine flexion and sustained loading. Brinckmann et al. (1988) also showed repeated compression loading can cause the spinal motion segments to fail at moderate loads. As indicated in Figure 5, the National Institute of Occupational Safety and Health has set a lower limit to protect most workers at 760 pounds $(3400 \mathrm{~N})$ of compression (Water et al., 1993).

A comparison of predicted compression forces with the NIOSH limits (which includes an upper Maximum Permissible Limit from 1981) is given in Figure 6. This clearly shows the importance of keeping heavier loads close to the body with an erect posture to minimize the spinal compression forces.

Because the earlier models of the spine were only for coplanar, sagittal plane evaluations, and many exertions are asymmetric three dimensional, a more complete model was needed. When an asymmetric exertion (e.g., one handed force, or twisted or laterally bent torso is being analyzed) many different torso muscle actions and passive supporting tissue reactions need to be considered. The first step in such a procedure requires that the position, orientation, cross-sectional size, and length of the various connective tissues be modeled at the lumbar spinal level. A geometric torso model proposed by Nussbaum and Chaffin (1996) for this purpose is shown in Figure 7. This model includes estimates of specific tissue geometry acquired from various CT scans (Chaffin et al., 1990; Moga 

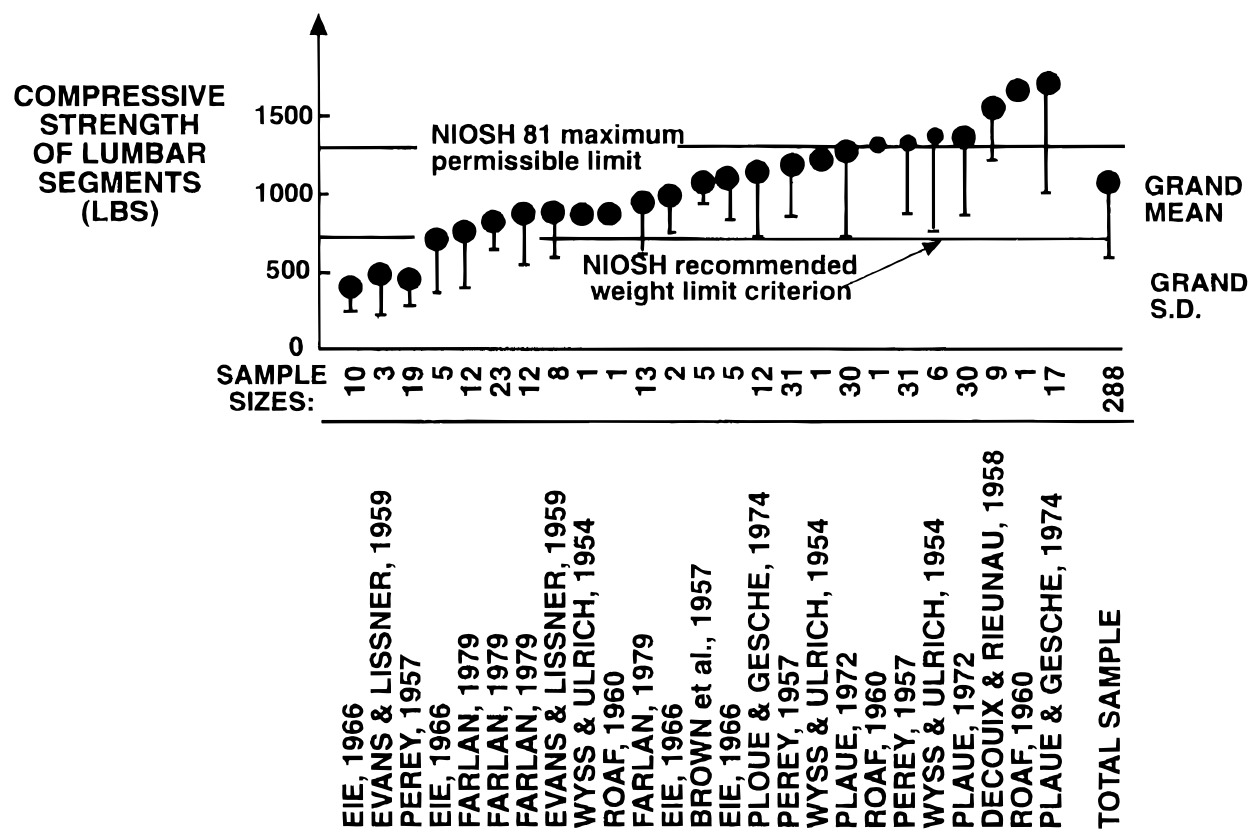

Figure 5 Composite of cadaver studies to determine the compression strengths of lumbar segments (adapted from Jäger, 1987).

et al., 1993; Tracy et al., 1989) along with passive tissue reaction forces estimated by McCully and Faulkner (1983), Nachemson, Schultz, and Berkson (1979), Miller et al. (1986), and others.

The most important predictors of spinal column stress, however, are the muscle reaction forces required to stabilize the spine when subjected to external load moments. In the 3D torso models various approaches have been used to predict the required reactive muscle forces. Perhaps the most commonly cited torso biomechanical model for 3D Static Analysis is that developed by Schultz and Andersson (1981). It is depicted in Figure 8. A revised version of this model has been developed by Bean, Chaffin, and Schultz (1988). This latter model provides a more efficient computational method for solving the linear programs used

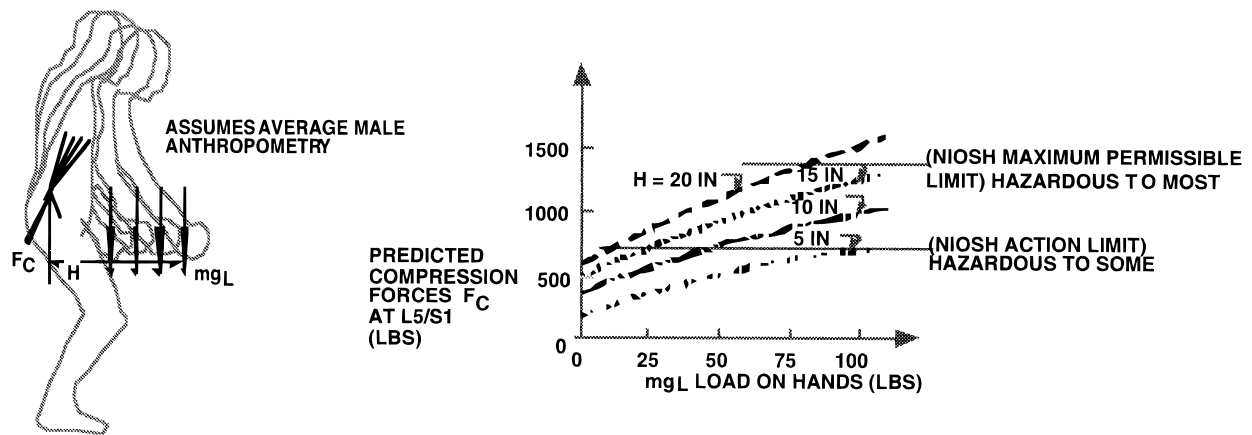

Figure 6 Predicted L5/S1 disc compression forces for varying loads lifted in four different positions from body (Chaffin and Anderson, 1991). 


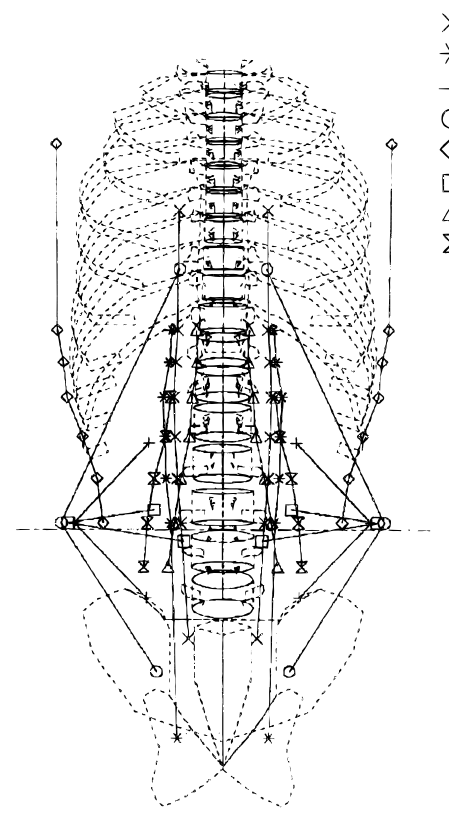

POSTERIOR VIEW $\times$ Erector Spinae

* Rectus Abdom.

+ Int. Oblique

O Ext. Oblique

$\diamond$ Lat. Dorsi

Trans. Abdom.

$\triangle$ Psoas

8 Quad. Lumborum

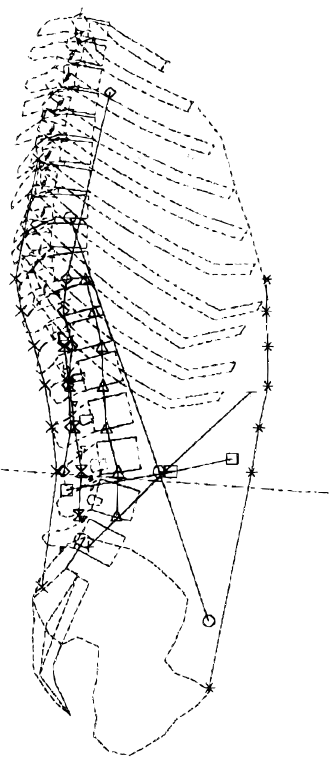

RIGHT-SIDE VIEW

Figure 7 Muscle geometry illustrated for a 50th percentile male. Muscles are treated as pointwise connections from origin to insertion. An imaginary cutting plane which bisect the L3/L4 motion segment is also shown (Nussbaum and Chaffin, 1996).

to simultaneously minimize the torso muscle contraction intensities and motion segment compression forces. The present model predicts the minimum muscle force contractile intensities required to meet the moment equilibrium requirements about the three orthogonal axis-of-rotation of the spinal motion segment. Given a set of optimal forces so computed, the model further seeks to minimize the disc compression forces. Because such an approach attempts to minimize both muscle intensity requirements and disc compression forces simultaneously, it is referred to as a "double linear optimization" approach.

More recently, Hughes and Chaffin (1995) proposed that a nonlinear objective function be used as the basis for selecting the various muscle reaction forces during a given exertion. They referred to this as the sum of the cubed muscle intensity objective. Nussbaum, Chaffin, and Martin (1996) also have proposed a neural network model to predict torso muscle actions. And most recently, Raschke and Chaffin (1996) have proposed that the external moment is normally distributed about the torso, and activates several muscles simultaneously depending on the direction and magnitude of the external moment.

\section{VALIDATION OF STRENGTH AND BACK FORCE PREDICTION MODELS}

The validation of the static strength predictions from the 2D and 3D static strength prediction models has been accomplished in three different studies. All three validations required using the models to simulate whole-body exertions and compare the percent 


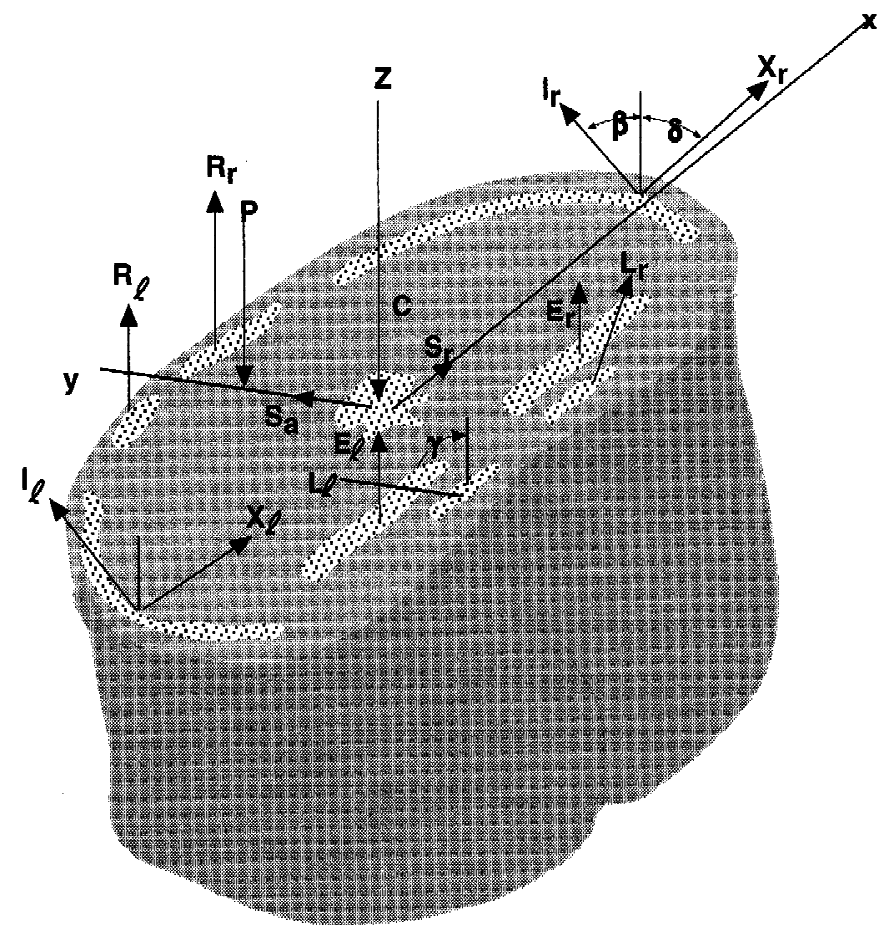

Figure 8 Schematic of 10-muscle model developed by Schultz and Andersson (1981).

capable predictions with the mean, 10th and 90th percentile strengths of a group of volunteers who performed the same tasks.

In the first validation Garg and Chaffin (1975) had 71 male Air Force personnel perform 38 different maximum arm exertions (i.e., lifts, pushes, pulls, etc.) in a variety of arm/torso postures while seated. They found the predicted strengths were highly correlated with the group strengths when performing the 38 upper body tasks ( $r=0.93$ to 0.97). Chaffin, Freivalds, and Evans (1987) simulated 15 different whole-body exertions in the sagittal plane that were also performed by both men and women volunteers from a variety of industries. In some of these tests over 1000 people performed the exertions, though on average about 200 people performed each. Comparison with the 2DSSPPTM program with the group strength data revealed a very high correlation $(r=0.92)$. This same study also included simulations with the 3DSSPPTM program of 72 different one arm exertions performed by five male army personnel. The correlations ranged from $r=$ 0.71 to 0.83 . Unfortunately, in this latter comparison, exact postural and bracing conditions were not available to use in the simulations. This and the small sample $(N=5)$ may have contributed to the lower correlations.

The last validation involved simulations of 56 one- and two-handed, whole-body exertions in 14 different symmetric, bent and twisted-torso postures (Chaffin and Erig, 1991). The simulation results were compared with the group strengths of 29 young males. Photographs from several views were available to assist in replicating the postures used by these subjects. The results indicated that if care is taken to assure that the postures used in the model simulation are the same as that chosen by people performing the exertions, the prediction error standard deviation will be less than $6 \%$ of the mean predicted value. 
In conclusion, it appears that the strength prediction models and population norms used in the present models are accurate in predicting the percentage of the population capable of performing a large variety of different types of maximal static exertions. One caution should be noted, however. At present the strength norms used as joint moment limits in the models are based on male and female populations who are relatively young (i.e., 18-49 years). To improve the models further, strength values are currently being gathered on older populations by this investigator. In this regard, one comparison involving 98 men and women with a mean age of 73 years showed a major decrease in strength performance in certain muscle functions. When these decreases were included in the 3DSSPPTM population data base, it was found that some exertions that could easily be performed by younger people were predicted to be impossible to perform by most older people (Chaffin et al., 1994).

Validation of the low back biomechanical model has been largely dependent on EMG estimates of muscle reactions in subjects performing controlled torso exertions. Hughes et al. (1994) discuss this procedure, and the results of comparisons with four different optimization procedures used to predict torso muscle responses to different external torso moment loads. Generally speaking, relatively high correlation $(r>0.8)$ are achieved when loading the torso approximately in the sagittal plane. With greater asymmetric or sudden loading, more complex muscle patterns result, sometimes with a 10-30\% antagonistic type of muscle response. These complex responses are often not well predicted $(r<0.6)$ by existing models. Thus, it is expected that the existing optimization based models may underpredict the muscle-induced compression and shear forces on the spinal motion segments by as much as $30 \%$, especially during sudden (i.e., jerking) motions, or lateral, asymmetric exertions. The newer, neural network and/or geometric moment distribution models are yet to be thoroughly tested under complex loading conditions. They may be less sensitive to this co-contraction phenomenon than existing optimization models.

\section{USABILITY OF STRENGTH AND LOW BACK PREDICTION MODELS FOR JOB DESIGN}

It should be clear from the preceding that there exists static strength and low back compression force prediction models that can be used to evaluate manual exertions. What follows is a description of the work necessary to make these models useful when performing job evaluations during the early phases of a job design process. To make these biomechanical models useful in this context required that the models be easy to implement and use on personal computers. Over the last 25 years several research engineers and computer programmers at the University of Michigan's Center for Ergonomics have worked to accomplish this goal, which resulted in the first 2-D Static Strength Prediction Program ${ }^{\mathrm{TM}}$ being licensed in 1984 by the University's Intellectual Property Office. This was followed in 1989 by the first 3-D Static Strength Prediction Program ${ }^{\mathrm{TM}}$ being licensed.

The main screen of the 2DSSPPTM program is depicted in Figure 9. The input values, (i.e., body link angles, hand forces, and anthropometry) are shown in the upper left quadrant. A stick figure depicting the body posture, the hand location, and the hand force direction that are used as inputs is depicted in the upper right quadrant. The predicted percent of the male and female population having sufficient strength to perform the designated exertion (in this case lifting a 44-pound stock reel) is shown in tabular and graphical form in the lower left quadrant. The back compression force predictions for a man and women performing the 44-pound lift is shown in the lower right quadrant. In this 


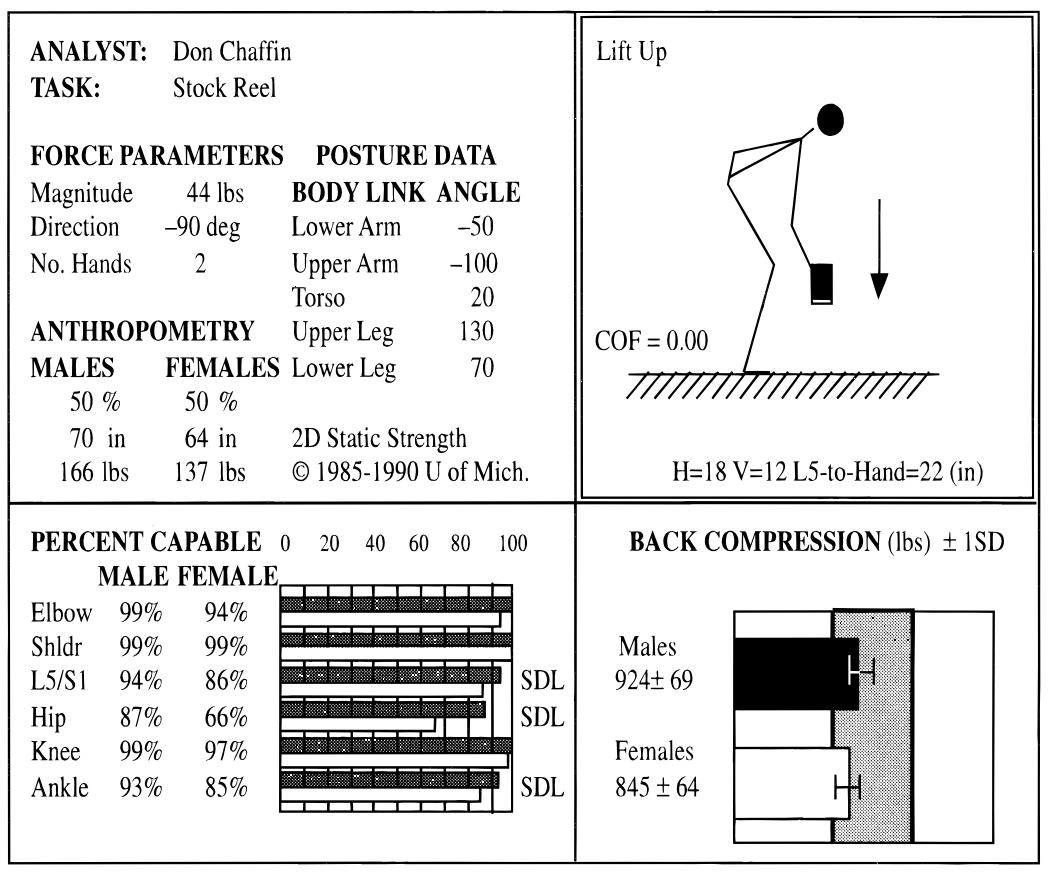

Figure 9 Main screen from the University of Michigan 2D Static Strength Prediction Program ${ }^{\mathrm{TM}}$ for personal computers (from the University of Michigan, IPO Software, Ann Arbor, MI 48109).

situation, from inspection of the percent capable predictions (left bottom), it is obvious that hip strengths are the most limiting muscle group strengths (only $66 \%$ of women and only $87 \%$ of men have sufficient hip strength to lift the 44-pound reel). Incidently, these values are below that recommended by NIOSH, which believes that jobs should accommodate $99 \%$ of men's and $75 \%$ of women's strength (or $90 \%$ of a mixed gender population). It also is shown in the right bottom quadrant that the L5/S1 compression forces of 924 and 845 pounds are above that recommended by NIOSH of 770 pounds.

The emphasis on this $2 \mathrm{D}$ version of the model was to provide a simple to use, and very fast job evaluation and design tool. Because only five body angles are required, along with a single hand force magnitude and direction, the analyst can easily modify input data and visualize the effects via changes in the stick figure configuration, percent capable graphs, back compression force graphs, foot coefficient of friction values, and body balance warnings. Though many iterations of joint angles and hand forces may be necessary to solve a particular exertion problem, the computational speed (less than $1 \mathrm{~s}$ on a slow 286 processor) has allowed many different users to benefit from this simple tool. It also has been used in conjunction with a video-based postural measurement system to evaluate sequential static exertions, and dynamic exertions (Liftrak, 1991).

The $3 \mathrm{DSSPP}^{\mathrm{TM}}$ program requires more input data than the 2D version depicted in Figure 9. Three-dimensional exertions often involve different forces on each hand that can act in any direction. Also, a model of the human body in 3D has 12 body links (some with three postural angles each to specify). Two types of input presentations have been used to assist in assuring the correct input data are used for 3D analyses. One presentation has three orthogonal views of a stick figure, and the other presents a shaded, enfleshed hominoid, which can be viewed from any direction. Figure 10 depicts these two presentations. 


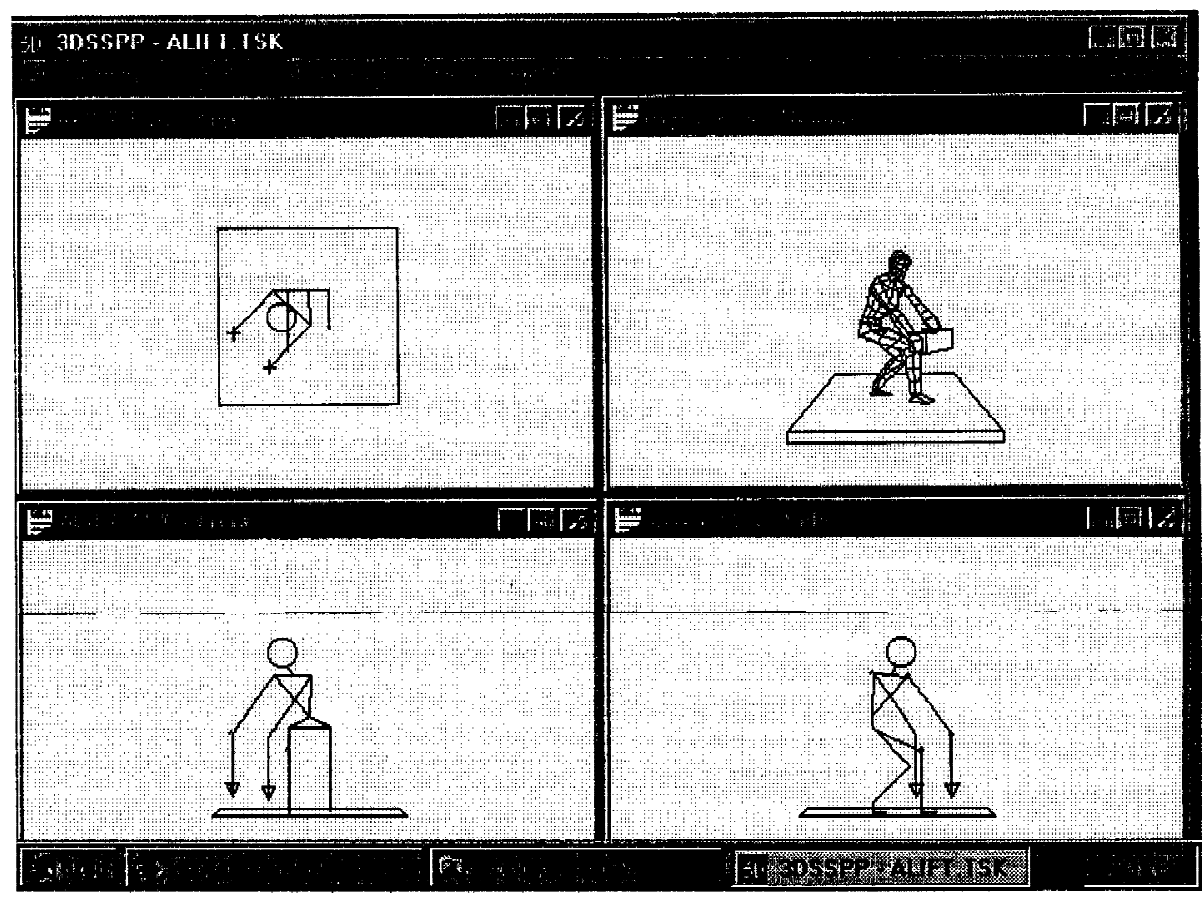

Figure 10 Input screen used in 3DSSPPTM to assure postural data are correct. Three orthogonal view stick figures are combined with an enfleshed hominoid that can be viewed from different perspectives (with permission University of Michigan Regents).

Once a posture has been set, the size of the individual is chosen from a menu, and the hand forces on both hands are entered on the keyboard (the hand force directions can be designated by moving the vector arrows extending from each hand in Figure 10 by pointing a cursor and clicking a mouse). With the input data complete, the necessary biomechanical computations are executed within a second of CPU time on most personal computers. The output is presented in both graphical and tabular form, with a summary table similar to that on the bottom of Figure 9 for the 2DSSPPTM program.

As discussed earlier, because the 3D program provides a user with so much flexibility in choosing the correct posture (i.e., strongest or minimum stress posture), without some assistance the user may choose suboptimal postures for high exertion tasks. In fact, Chaffin and Erig (1991) found that a $10^{\circ}$ error in specifying the angle of a joint that was associated with the most limiting muscle strengths could produce a $30 \%$ error in the resulting population strength prediction. Beck (1992) also found that it could take $11 \mathrm{~min}$ to manipulate the stick figures shown in Figure 10 so that they accurately duplicated postures shown in photographs to users.

To improve the usability of the 3DSSPPTM Beck (1992) developed the enfleshed hominoid, which can easily be rotated and enlarged or shrunk to assist a user in visualizing a particular posture of interest. Perhaps more important though was the addition of an inverse kinematic structure that maintains the integrity of the linkage system as the user moves the hands or body joints around in space. The actual repositioning of the hominoid is accomplished by pointing a cursor at a joint and moving it with a mouse. The inverse kinematic structure also assists the user in choosing initial feasible postures by referring 
to behavioral data about preferred body postures. The behavioral-based inverse kinematic method of predicting initial postures is based on previous work by Kilpatrick (1970), Snyder, Chaffin, and Schultz (1972), and Park (1973) wherein different subjects were photographed while assuming a large variety of postures. The digitized photographs were used to generate prediction equations which relate $\mathrm{X}, \mathrm{Y}, \mathrm{Z}$ coordinates of body reference points to hand locations while the subjects were in their "preferred postures." Beck used these equations as the basis for choosing body linkage configurations required to set the hands in a specific location. By using an inverse kinematic structure, as a particular segment is moved the other segments in the kinematic chain move to the degree necessary to keep the hands in their original location. Imposing this structure not only allowed the computer program to find feasible and preferred postures, but also allows the analyst to point to a segment and move it to another location without losing a desired hand location.

Beck (1992) was able to show that by using both the enfleshed hominoid and the behavioral inverse kinematic posture prediction scheme, novice job analysts were able to reduce their postural entry time by $57 \%$, and were $11 \%$ more accurate than using the existing data entry scheme. Thus, the inclusion of inverse kinematics, behaviorally based posture prediction methods, and improved human form graphics have made the use of these models relatively easy for job design evaluations. By simply clicking and pointing to parts of the body, one can adjust postures and hand forces and have a comprehensive biomechanical assessment of a specific exertion without the need to mock-up a workplace and have subjects demonstrate different methods of performing a manual exertion. This design evaluation capability is demonstrated in Figure 11, where two different methods of lifting a load from the floor are simulated.

\section{DISCUSSION}

When designing equipment today, various types of computer programs are often used to assist in the structural evaluation of proposed designs. Structural analysis using kinematic and FEM models are routinely performed to avoid the high costs and delays incurred when it is required to build and test a prototype during the design process. Unfortunately, simulations of humans physically interacting with proposed hardware systems have been restricted mostly to problems associated with human reach, fit, and visualization. The ability to consider the normal anthropometric variations in the size of a population during the design of a job or product is certainly commendable, because human size may vary by 1.6:1 in a normal population. But with so many industrial jobs still requiring significant physical strengths, which vary by 10:1 in the normal population, it is imperative that job design assess the potential impact of a mismatch in this human attribute.

This article has proposed that the technology needed to predict human population static strengths is well developed, has a known confidence interval, and can be easily utilized on existing personal computer platforms. Further, existing job oriented CAD systems now being used for workplace layout designs can easily import the human strength models discussed in this article. This is demonstrated in Figure 12 with an AutoCad ${ }^{\mathrm{TM}}$ workplace simulation, wherein a worker is depicted while performing manual exertions in an automotive assembly task. In this case the $3 \mathrm{DSSPP}^{\mathrm{TM}}$ program is directly linked to the AutoCad ${ }^{\mathrm{TM}}$ files depicting the workspace, thus enabling changes in the workplace/ equipment to be evaluated quickly by the $3 \mathrm{DSSPP}^{\mathrm{TM}}$ program. 


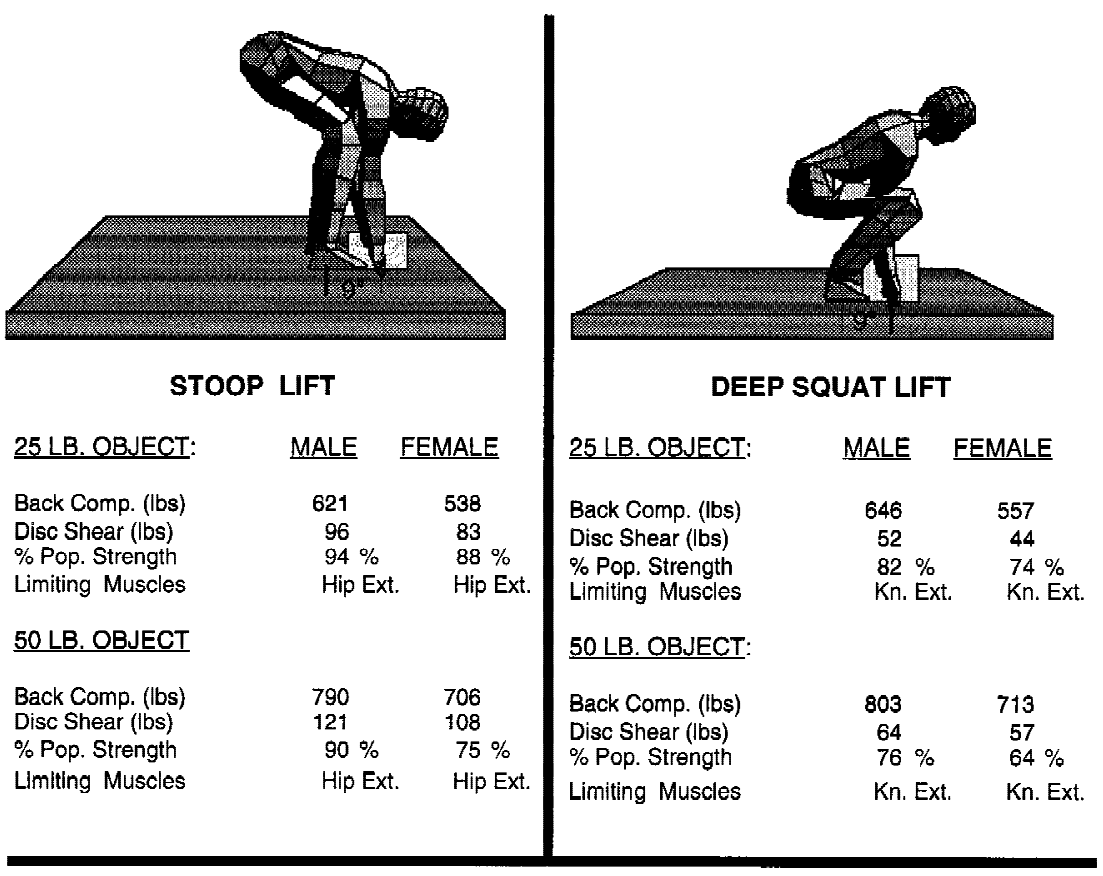

Figure 11 Comparison of two different postures used to lift 25- and 50-pound objects from the floor close to feet using the Michigan 3DSSPPTM. Even though the hand locations are the same for each posture, and the back compression forces do not change much, the stoop lift makes better use of the powerful hip extensor muscles and thus allows more people to perform the lift than is the case in the deep squat lift wherein the weaker knee extensors limit more people.

Clearly, the existing biomechanical models are not complete nor accurate for all types of exertions. Where fast movements are required combined with maximum strength exertions the static models will overpredict the population's capabilities. Where it is difficult to grip an object being manipulated, the models will overpredict the population's capabilities. Where older populations are employed, the models will overpredict the populations' capabilities. These are issues that are being addressed by this and other biomechanics researchers. As improved biomechanical models and population data bases are developed these will be incorporated in subsequent versions of the 3DSSPPTM.

What is important now is to realize that a great deal of ergonomic information regarding population variability in strength and low back failure is accessible in a form that can improve future job designs. It is hoped that this article will assist those interested in understanding the rapidly developing technologies needed to further improve manual tasks in industry.

\section{ACKNOWLEDGMENT}

The author wishes to acknowledge Ford Motor Company for their financial support of this work, and in particular Dr. Bradley Joseph and Mr. Glenn Jimmerson. Also, I wish to thank Robert Feyen and Randy Rabourn for assisting in preparation of this article. 


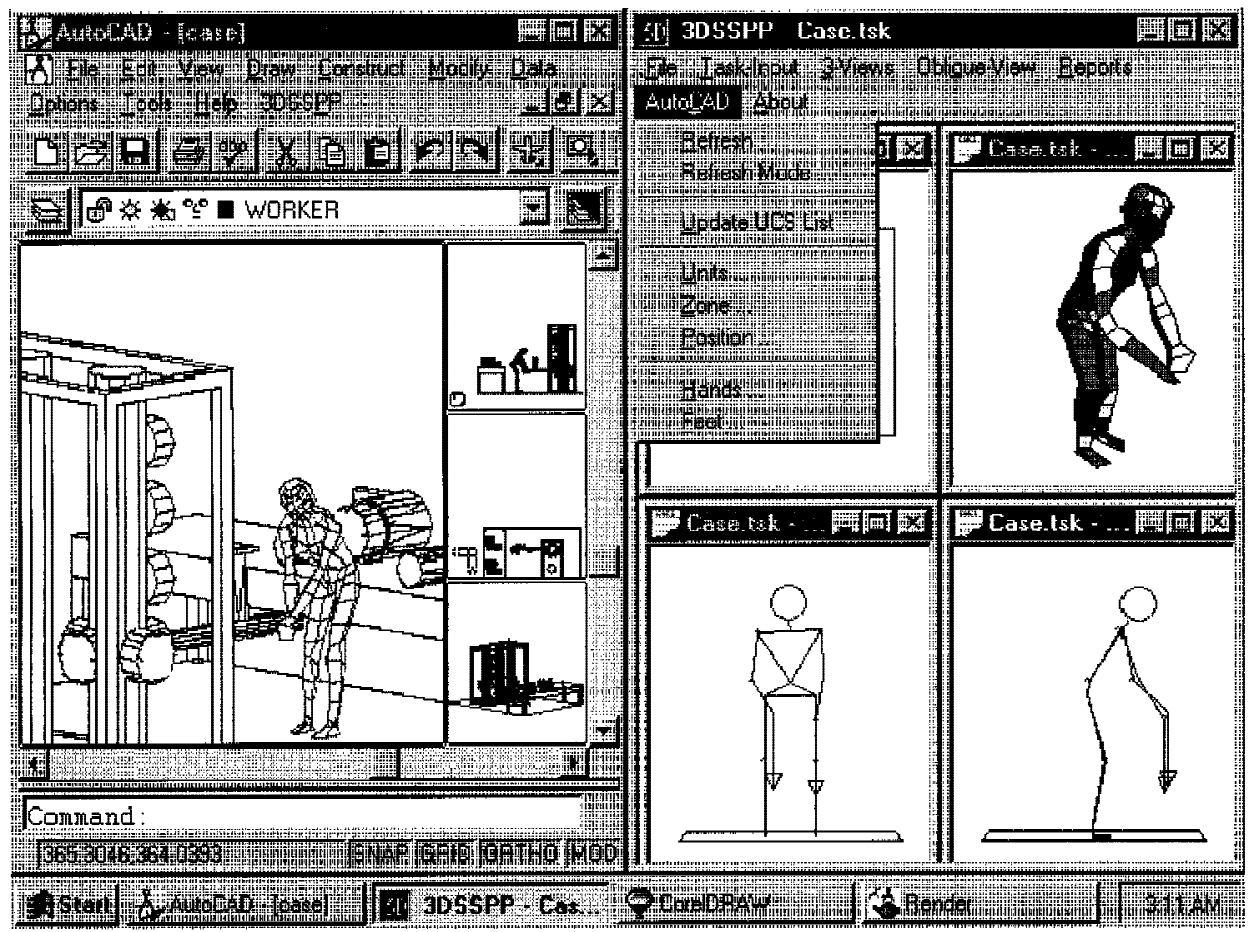

Figure 12 Depicts how AutoCad ${ }^{\mathrm{TM}}$ rendering of workplace can be combined with 3DSSPPTM to assess effects of workspace/equipment changes on population strengths.

\section{REFERENCES}

Adams, M.A. and Dolan, P., 1995, Recent advances in lumbar spinal mechanics and their clinical significances, Clinical Biomechanics, 10(1), 3-19.

Ayoub, M.M. and El-Bassoussi, M.S., 1978, Dynamic biomechanical model for sagittal plane lifting activities. In Safety in Manual Materials Handling by C.G. Drury (Ed.) (Cincinnati: National Institute of Occupational Safety and Health), pp. 78-185.

Badler, N.L., Becket, W.M., and Webber, B.L, 1995, Simulation and analysis of complex human tasks for manufacturing. In Proceedings of SPIE-The International Society for Optical Engineering, 2596, pp. 225-233.

Bean, J.C., Chaffin, D.B., and Schultz, A.B, 1988, Biomechanical model calculation muscle contraction forces: A double linear programming method, Journal of Biomechanics, 21(1), 59-66.

Beck, D.J., 1992, Human factors of posture entry into CAD systems. Unpublished doctoral dissertation, University of Michigan, Industrial and Operations Engineering, Ann Arbor, MI.

Brinckmann, P., Frobin, W., Hierholzer, E., and Horst, M., 1983, Deformation of the vertebral endplate under axial loading of the spine, Spine, $\mathbf{8}, 851$.

Burggraff, J.D., 1972, An isometric biomechanical model for sagittal plane leg extension. Unpublished master's thesis, University of Michigan, Ann Arbor, MI.

Chaffin, D.B., 1969, A computerized biomechanical model: developmental and use in studying gross body actions, Journal of Biomechanics, 2, 429-441.

Chaffin, D.B., 1975, On the validity of biomechanical models of the low back for weight-lifting analysis. In Proceedings of ASME (75-WA-Bio-1) American Society of Mechanical Engineers, New York, pp. 1-12.

Chaffin, D.B. and Andersson, G.B.J. (eds.), 1991, Occupational Biomechanics (John Wiley \& Sons, Inc., New York). 
Chaffin, D.B. and Baker, W.H., 1970, A biomechanical model for analysis of symmetric sagittal plane lifting, AIIE Transactions, 2(1), 16-27.

Chaffin, D.B. and Erig, M., 1991, Three-dimensional biomechanical static strength prediction model sensitivity to postural and anthropometric inaccuracies, IIE Transactions, 23(3), 215-227.

Chaffin, D.B., Freivalds, A., and Evans, S.M., 1987, On the validity of an isometric biomechanical model of worker strengths, IIE Transactions, 19(3), 280-288.

Chaffin, D.B., Redfern, M.S., Erig, M., and Goldstein, S.A., 1990, Lumbar muscle size and location measurements from CT scans of 96 older women, Clinical Biomechanics, 5(1), 9-16.

Chaffin, D.B., Woolley, C.B., Buhr, T., and Verbrugge, L., 1994, Age effects in biomechanical modeling of static lifting strengths. In Proceedings of Human Factors Society, Nashville, TN.

Clarke, H.H., 1966, Muscle Strength and Endurance in Man (Prentice-Hall, Englewood Cliffs, NJ).

Clauser, C.E., McConville, J.T., and Young, J.W., 1969, Weight, volume and center of mass of segments of the human body, AMRL-TR-69-70, Aerospace Medical Research Laboratories, WrightPatterson AFB, Ohio.

Dempster, W.T., 1955, Space requirements of the seated operator, WADC-TR-55-159, Aerospace Medical Research Laboratories, Wright-Patterson AFB, Ohio.

Drillis, R. and Contini, R., 1966, Body Segment Parameters, BP174-945, Tech. Rep. No. 1166.03, S. of Engineering and Science, New York University, New York.

Fortin, C., Gilbert, R., Beuter, A., Laurent, F., Schiettekatte, J., Carrier, R., and Dechamplain, B., 1990, SAFEWORK: A microcomputer-aided workstation design and analysis. New advances and future developments. In Computer-Aided Ergonomics by W. Karwowski, A.M. Genaidy, and S.S. Asfour (eds.) (Taylor and Francis, London).

Freivalds, A., Chaffin, D.B., Garg, A., and Lee, K.S., 1984, A dynamic biomechanical evaluation of lifting maximum acceptable loads, Journal of Biomechanics, 17, 251-262.

Garg, A.D. and Chaffin, D.B., 1975, A biomechanical computerized simulation of human strength, AIIE Transactions, 7(1), 1-15.

Grobelny, J., Cysewski, P., Karwowski, W., and Zurada, J., 1992, APOLIN: A 3-dimensional ergonomic design and analysis system. In Computer Applications in Ergonomics, Occupational Safety and Health by M. Mattila, and W. Karwowski (eds.) (Elsevier Science Publishers BV, Amsterdam).

Hoekstra, P.N., 1993, Some uses of active viewing in computer aided anthropometric assessment. In Designing for Diversity Proceedings of the Human Factors and Ergonomics Society, vol. 1, pp. 494-498.

Hughes, R.E. and Chaffin, D.B., 1995, The effect of strict muscle stress limits on abdominal muscle force predictions for combined torsion and extension loadings, Journal of Biomechanics, 28(5), 527-533.

Hughes, R.E., Chaffin, D.B., Lavender, S.A., and Andersson, G.B.J., 1994, Evaluation of muscle force prediction models of the lumbar trunk using surface electromyography, Journal of Orthopometric Research, 12, 689-698.

Jäger, M., 1987, Biomechanisches Modell des Menschen zur Analyse und Beurteilung der Belastung der Wirbelsäule bei der Handhabung von Lasten, VDI Verlag, Reihe 17 Biotechnik Nr. 33.

Kilpatrick, K.E., 1970, A model for the design of manual workstations. Unpublished doctoral dissertation, University of Michigan, Ann Arbor, MI.

Launis, M. and Lehtela, J., 1990, Man models in the ergonomic design of workplaces with the microcomputer. In Computer-Aided Ergonomics by W. Karwowski, A.M. Genaidy, and S.S. Asfour (eds.) (Taylor and Francis, London).

Leskinen, T.P., Stålhammar, H.R., Kuorinka, I.A., and Troup, J.D.G., 1983, The effect of inertial factors on spinal stress when lifting, Engineering in Medicine, 12(2), 87-89.

Liftrak, 1991, Motion Analysis Corporation, 3650 North Laughlin Road, Santa Rose, CA 95403.

Martin, J.B. and Chaffin, D.B., 1972, Biomechanical computerized simulation of human strength in sagittal plane activities, AIIE Transactions, 4(1), 19-28.

McCully, K.K. and Faulkner, J.A., 1983, Length-tension relationship of mammalian diaphragm muscles, Journal of Applied Physiology, 54, 1681-1686.

McDaniel, J.M., 1990, Models for ergonomic analysis and design: COMBIMAN and CREW CHIEF. In Computer-Aided Ergonomics by W. Karwowski, A.M. Genaidy, and S.S. Asfour (eds.) (Taylor and Francis, London).

Miller, J.A.A., Schultz, A.B., Warwick, D.N., and Spencer, D.L., 1986, Mechanical properties of lumbar spine motion segments under large loads, Journal of Biomechanics, 19, 79-84. 
Mollard, R., Ledunois, S., Ignazi, G., and Coblentz, A., 1992, Researches and developments on postures and movements using CAD techniques and ERGOTDATA. In Computer Applications in Ergonomics, Occupational Safety and Health by M. Mattila and W. Karwowski (eds.) (Elsevier Science Publishers BV, Amsterdam).

Moga, P.J., Erig, M., Chaffin, D.B., and Nussbaum, M.A., 1993, Torso muscle moment arms at intervertebral levels T10 through L5 from CT scans on eleven male and eight female subjects, Spine, 18(15), 2305-2309.

Morris, J.M., Lucas, D.B., \& Bressler, B., 1961, Role of the truck in stability of the spine, Journal of Bone and Joint Surgery, 43A, 327-351.

National Institute for Occupational Safety and Health, 1981, A Work Practice Guide for Manual Lifting, Technical Report No. 81-122, U.S. Department of Health and Human Services (NIOSH) (Cincinnati, OH).

Nachemson, A.L. and Elfstrom, G., 1970, Intravital dynamic pressure measurements in lumbar disc, Scandinavian Journal of Rehabilitation Medicine, Suppl. 1, 1-39.

Nachemson, A.L., Schultz, A.B., and Berkson, M.H., 1979, Mechanical properties of human lumbar spine motion segments: influences of age, sex, disc level, and degeneration, Spine, 4, 1-8.

Nayar, N., 1995, Deneb/ERGO-a simulation based human factors tool. In Proceedings of the Winter Simulation Conference.

Nussbaum, M.A. and Chaffin, D.B., 1996, Development and evaluation of a scalable and deformable geometric model of the human torso, Clinical Biomechanics, 11(1), 25-34.

Nussbaum, M.A., Chaffin, D.B., and Martin, B.J. 1996, A back-propagation neural network model of lumbar muscle recruitment during moderate static exertions, Journal of Biomechanics, 28(9), $1015-1024$.

Park, K., 1973, A computerized simulation model of postures during manual materials handling. Unpublished doctoral dissertation, University of Michigan, Ann Arbor, MI.

Porter, J.M., Freer, M., Case, K., and Bonney, M.C., 1995 Computer aided ergonomics and workspace design. In Evaluation of Human Work: A Practical Ergonomics Methodology by J.R. Wilson and E.N. Corlett (eds.) (Taylor and Francis, London).

Raschke, U. and Chaffin, D.B., 1996, Trunk and hip muscle recruitment in response to external anterior lumbosacral shear and moment loads, Clinical Biomechanics, 11(3), 145-152.

Schanne, F.A., 1972, A three dimensional hand force capability model for the seated operator. Unpublished doctoral dissertation, University of Michigan, Ann Arbor, MI.

Schultz, A.B. and Andersson, G.B., 1981, Analysis of loads on the lumbar spine, Spine, 6(1), 7682.

Snyder, R., Chaffin, D., and Schultz, R., 1972, Link system of the human torso, HSRI report 71112, Highway Safety Research Institute, and University of Michigan, Ann Arbor, MI and AMRLTR-71-88, Aerospace Medical Research Laboratories, Ohio.

Tracy, M.F., Gibson, M.J., Szypryt, E.P., et al., 1989, The geometry of the muscles of the lumbar spine determined by magnetic resonance imaging, Spine, 14, 186-193.

U.S. Department of Labor, Bureau of Labor Statistics, 1996, Characteristics of Injuries and Illnesses Resulting in Absences from Work, 1984 (Washington, DC).

Waters, T.R., Putz-Anderson, V., Garg, A., and Fine, L.J., 1993, Revised NIOSH equation for the design and evaluation of manual lifting tasks, Ergonomics, 36(7), 749-776.

Westerink, J., Tragter, H., Van Der Star, A., and Rookmaaker, D.P., 1990, TADAPS: a threedimensional CAD man model. In Computer-Aided Ergonomics by W. Karwowski, A.M. Genaidy, and S.S. Asfour (eds.) (Taylor and Francis, London). 